\title{
Explicit Associations with Autism and Disability
}

\author{
Jennifer L. Stevenson, PhD, ${ }^{1}$ and Theresa G. Mowad, BS ${ }^{2}$
}

\begin{abstract}
Objectives: Autistic people call for greater acceptance even though the general public has greater awareness of the autism spectrum. This study investigated explicit or conscious attitudes toward the autism spectrum and disability in college students and the general population. We hypothesized that both samples would associate "a person on the autism spectrum" with more negative attributes than other types of people except for "a person with a disability."

Methods: In Phase 1, participants generated 10 word associations for 8 labels: a person on the autism spectrum, a person not on the autism spectrum, a person with a disability, a person without a disability, a college student, a professor, a child, and a parent. In Phase 2, participants rated the 10 most common words for each label (type of person) in Phase 1 on a 7-point Likert scale from extremely negative to extremely positive. Ninety-nine undergraduate students and 106 adults recruited through Amazon Mechanical Turk completed Phase 1. One hundred twenty-two undergraduate students and 101 adults recruited through Amazon Mechanical Turk completed Phase 2. Results: Only "a person with a disability" in the general population sample was rated as having significant negative associations. However, the associations of "a person with a disability" were rated more negatively than all other labels in both samples, and the associations of "a person on the autism spectrum" were rated as second most negative in the general population sample.

Conclusion: Explicit associations toward disability and autism were somewhat mixed. Adults in the general population tended to have more negative explicit associations with disability, and to a lesser extent autism. These results underscore the need to examine attitudes in samples more representative of the general population. Furthermore, evidence of possible explicit negative associations is concerning and highlights the imperative need to confront ableism.
\end{abstract}

Keywords: autism, disability, explicit attitudes

\section{Lay Summary}

Why was this study done?

The general public claims to be aware of the autism spectrum and recent research suggests that they have greater knowledge about the autism spectrum than they did in the past. However, as autistic individuals have articulated, autism awareness is not the same as autism acceptance. In order for autistic individuals to be fully included into society, we must move to autism acceptance. One way researchers examine potential discrimination is by studying attitudes.

What was the purpose of this study?

This study examines people's explicit attitudes toward the autism spectrum and to disability. Explicit attitudes are attitudes that are conscious and controllable.

What did the researchers do?

Two groups of adults participated in the study: a group of college students and a noncollege sample of adults designed to better represent the general population of adults. Participants completed an online study wherein

\footnotetext{
${ }^{1}$ Department of Psychology, Ursinus College, Collegeville, Pennsylvania.

${ }^{2}$ Center for Advanced Retinal and Ocular Therapeutics, University of Pennsylvania, Philadelphia, Pennsylvania.
} 
they were asked to list word associations for different types of people including "a person on the autism spectrum" and "a person with a disability." In a second study, participants then rated the most common associations from extremely negative to extremely positive.

\section{What were the results of the study?}

The results were somewhat mixed. Only the set of associations of "a person with a disability" were rated by participants in the noncollege group as negative. However, the associations of "a person with a disability" were rated more negatively than all other types of people in both groups, and the associations of "a person on the autism spectrum" were rated as second most negative in the noncollege sample.

\section{What do these findings add to what was already known?}

The majority of research on explicit attitudes toward autism has focused on children, including how interventions may improve attitudes toward autistic children. Therefore, this research provides much needed information on the state of attitudes toward autistic individuals more generally. This research also provides a comparison of attitudes toward autism, disability, and other groups. Furthermore, research assessing attitudes toward autism in adults has largely focused on college students, whereas this research considered both college students and a noncollege sample.

\section{What are the potential weaknesses in the study?}

These findings may not extend to a more diverse population as both groups had relatively high education levels, were primarily White non-Hispanic and were living in the United States. Furthermore, participants may have generated more positive associations for "a person on the autism spectrum" and "a person with a disability" because they wanted to be viewed in a favorable light. This is known as a social desirability bias.

How will these findings help autistic adults now or in the future?

Unfortunately, possible explicit negative associations with the autism spectrum and with disability are concerning as they reflect people's conscious and controllable attitudes. These results highlight a need for action and also support autistic individuals' demand for actions toward autism acceptance.

\section{Introduction}

W ITH AN ENTIRE MONTH devoted to autism awareness, most of the general public is aware of the autism spectrum. In fact, at least $80 \%$ of adults report they are aware of the autism spectrum ${ }^{1,2}$ and an increased awareness does generally correspond to an increase in knowledge about the autism spectrum. ${ }^{1,3,4}$ However, as autistic self-advocates point out, awareness is not the same as acceptance..$^{5-8}$ Despite widespread awareness and increased knowledge of the autism spectrum, we have not reached an inclusive society for autistic individuals. ${ }^{9}$ Furthermore, we have not reached an inclusive society for disabled individuals, in general. ${ }^{10}$

One method to investigate whether autistic people experience discrimination assesses explicit (conscious) and implicit (unconscious) attitudes toward the autism spectrum. The majority of research on attitudes toward autism explores explicit attitudes toward autistic children either by adults ${ }^{1-14}$ or other children. ${ }^{13,15-19}$ The few research studies exploring explicit attitudes toward autistic adults consider either a parent's perspective toward an adult autistic child ${ }^{20}$ or college students toward autistic peers. ${ }^{21-25}$

Furthermore, research on attitudes toward autism also focuses on two questions: can attitudes change with intervention $^{16,18,19,26-29}$ and does providing the diagnostic status affect attitudes. ${ }^{11,12,19,24,30}$ Intervention research focuses almost exclusively on children ${ }^{16,18,19,26,29}$ and although some studies suggest even brief interventions can produce positive change in attitudes, ${ }^{16,18,27,28}$ other studies suggest attitudes toward autism may be resistant to change. ${ }^{19,26}$

The addition of diagnostic status often leads to more positive attitudes about autistic individuals ${ }^{11,12,21,30}$; however, this is not always the case. ${ }^{19,22,24}$ For instance, one study manipulated the degree of autistic characteristics and the presence or absence of a diagnostic label in vignettes to determine whether autistic traits or diagnostic labels affect attitudes, specifically, stigmatizing attitudes in adults. They found that it was the degree of autistic traits, not the presence of a diagnostic label, that changed adults' self-reported attitudes. ${ }^{22}$

Novel research on explicit attitudes toward autistic individuals considers first impressions. Specifically, neurotypical children and adults form negative first impressions of autistic peers, leading neurotypical individuals to be less willing to interact with autistic individuals. ${ }^{30,31}$ After viewing stimuli (e.g., videos) that are either labeled or unlabeled with diagnostic information, participants rate the person on a series of adjectives. This method improves on past research because both the effect of the diagnostic label and differences between diagnostic conditions (e.g., autism, no diagnosis, and schizophrenia ${ }^{30}$ ) can be compared.

This study assessed explicit attitudes toward the autism spectrum and disability in two different samples: undergraduate students and a general population sample of adults to examine whether explicit attitudes are similar across these two groups. Moreover, this study used a different methodology to assess explicit attitudes. Instead of using a 
self-report rating scale, participants self-generated associations with the labels for a person on the autism spectrum (a specific disability), a person with a disability, a person not on the autism spectrum, a person without a disability, and four other comparison individuals in Phase 1, and rated the valence of the most common associations in Phase 2. Selfgenerated associations have been used to study disability ${ }^{32}$ and puzzle pieces as part of a study on autism puzzle piece logos ${ }^{33}$; however, the free responses have previously been coded by the researchers. This approach allowed us to examine the valence of explicit attitudes toward individuals on the autism spectrum compared with other groups of people instead of comparing change in attitudes due to an intervention $^{16,18,19,26-29}$ or change due to the presence of a diagnostic label. ${ }^{11,12,19,24,30}$ Comparing explicit attitudes for multiple groups of people is more in line with how implicit attitudes are assessed (e.g., comparing reaction times to a person with a disability versus a person without a disability when paired with positive and negative words; for a review see Ref. ${ }^{34}$ ). Furthermore, the online two-part method was designed to reduce social desirability bias that may be present when completing a self-report questionnaire. ${ }^{35}$ We hypothesized that participants would generate many common associations for "a person not on the autism spectrum" and "a person without a disability" due to the potential overlap in these two labels (e.g., participants might envision the same prototypical person). However, we hypothesized participants would generate few common associations for "a person on the autism spectrum" and "a person with a disability" because "a person on the autism spectrum" is a more specific example of "a person with a disability." We also hypothesized that both samples would associate "a person on the autism spectrum" with more negative attributes than other labels except for "a person with a disability."

\section{Methods}

\section{Phase 1}

Participants. Ninety-nine undergraduate students (76 female, 19 male, 4 nonbinary) between 18 and 22 years (mean $[M]=19.72$, standard deviation $[S D]=1.13$ ) and 106 adults recruited through Amazon Mechanical Turk (59 female, 47 male) between 21 and 65 years $(M=33.44$,
$S D=9.63$ ) completed Phase 1. Undergraduate students represented all class years, and general population adults on average had some college education $(M=14.75$ years, $S D=1.81$ ). Demographic information is provided in Table 1. We excluded an additional 31 undergraduate students and 18 general population adults from analysis because they either did not complete the study ( $n=26$ undergraduates and $n=6$ general population adults), they completed the study twice ( $n=3$ general population adults), or they did not give serious responses to all items ( $n=5$ undergraduates and $n=9$ general population adults).

Materials and procedure. The study was approved by and conducted in accordance with the Ursinus College Institutional Review Board. After giving informed consent and without receiving any information about the autism spectrum or disability, participants were asked to "please list the first 10 words (e.g., synonyms, adjectives, or other descriptors) that come to mind when you think of" "a person on the autism spectrum," "a person not on the autism spectrum,", "'a person with a disability," "a person without a disability,", "'a college student," "a professor," "a child," and "a parent." There was no time limit to complete the task and the list was provided in a pseudo-randomized order that was consistent for all participants. At the end of the study, participants completed a brief demographic questionnaire. All participants completed the study online using Qualtrics. Undergraduate students received academic credit in an introductory psychology course or a chance to win a gift card for their participation, and general population adults received $\$ 2$ for completing the study.

\section{Phase 2}

Participants. One hundred twenty-two undergraduate students (88 female, 31 male, 2 nonbinary) between 18 and 35 years $(M=20.21, S D=1.87)$ and 101 adults recruited through Amazon Mechanical Turk (50 female, 51 male) between 22 and 65 years $(M=35.97, S D=10.48)$ completed Phase 2. Undergraduate students represented all class years and general population adults on average had some college education $(M=14.64$ years, $S D=1.87)$. Demographic information is provided in Table 1 . We excluded an additional 31 undergraduate students and 37 general population adults

Table 1. Demographic Information for Both Samples in Phase 1 and Phase 2

\begin{tabular}{|c|c|c|c|c|}
\hline & \multicolumn{2}{|c|}{ Phase 1} & \multicolumn{2}{|c|}{ Phase 2} \\
\hline & Undergraduates & General population & Undergraduates & General population \\
\hline Gender: female/male/nonbinary & $76 / 19 / 4$ & $59 / 47 / 0$ & $88 / 31 / 2$ & $50 / 51 / 0$ \\
\hline Age: $M(S D)$ & $19.72(1.13)$ & $33.44(9.63)$ & $20.21(1.87)$ & $35.97(10.48)$ \\
\hline Hispanic/Latino: no/yes & $96 / 3$ & $94 / 12$ & $117 / 4$ & $89 / 12$ \\
\hline Race: White/person of color ${ }^{\mathrm{a}}$ & $81 / 18$ & $88 / 18$ & $102 / 19$ & $83 / 18$ \\
\hline Education level: median or $M(S D)$ & Sophomore & $14.75(1.81)$ & Sophomore & $14.64(1.87)$ \\
\hline $\begin{array}{l}\text { Autism connection: none/close friend } \\
\text { or family member/self-identify }\end{array}$ & $51 / 42 / 6$ & $84 / 20 / 2$ & $70 / 46 / 6$ & $80 / 17 / 4$ \\
\hline $\begin{array}{l}\text { Disability connection: none/close friend } \\
\text { or family member/self-identify }\end{array}$ & $43 / 40 / 16$ & $74 / 25 / 7$ & $63 / 43 / 16$ & $73 / 19 / 9$ \\
\hline
\end{tabular}

${ }^{\text {a}}$ Participants could self-identify their race as White, Black or African American, Asian, American Indian and Alaskan Native, Native Hawaiian and Other Pacific Islander, some other race, or more than one race.

$M$, mean; $S D$, standard deviation. 
from analysis because they either did not complete the study ( $n=30$ undergraduates and $n=6$ general population adults), they completed the study twice ( $n=1$ undergraduate and $n=1$ general population adult), or they failed at least one of three attention checks ( $n=30$ general population adults).

Materials and procedure. The study was approved by and conducted in accordance with the Ursinus College Institutional Review Board. After giving informed consent in which participants were told they were completing a "follow-up study examining people's associations with groups of people (e.g., college students, people on the autism spectrum, and people with disabilities)," participants rated the 10 most common words for each label (i.e., type of person) in Phase 1 on a 7-point Likert scale from extremely negative to extremely positive. Words were presented in pseudo-random order (consistent for all participants) in groups of five or six on a page. The general population sample completed ratings for three attention check words (i.e., positive, negative, and neutral). At the end of the study, participants completed a brief demographic questionnaire. All participants completed the study online using Qualtrics. Undergraduate students received academic credit in an introductory psychology course or a chance to win a gift card for their participation, and general population adults received $\$ 1$ for completing the study.

\section{Data analysis}

All analyses were conducted separately by sample unless otherwise noted.

Phase 1. Individual participants' responses were inspected for completeness and seriousness. Participants were excluded if they did not provide 10 serious associations for each label. Nonserious responses included writing a single letter, question marks, dashes, idk, or writing out a sentence or phrase across multiple associations.

Afterward, the associations were combined for each label to determine the most common associations. Participants' typos were ignored (e.g., independant was recoded as independent). In addition, the following rules were used to combine associations: (1) singular and plural words were combined as the singular word (e.g., kid and kids); (2) present tense and past tense of verbs were combined as the present tense of verbs (e.g., drink and drunk); (3) present tense of verbs and words ending in ing were combined as the ing form (e.g., love and loving); and (4) words that were virtually synonymous were combined (e.g., caregiver and caretaker; dad, daddy, and father; wise and wisdom). Multiple rules could apply (e.g., drink, drunk, and drinking were combined). The 10 most common associations for each label were used in Phase 2. If the 10th most common association was a tie, all responses as part of the tie were included.

The most common associations generated from Phase 1 were analyzed qualitatively to assess the associations in common between the different labels and the two samples.

Phase 2. Mean ratings were calculated for each individual association. In addition, mean ratings were calculated for the set of 10 most common associations for each label.
Qualitative analyses. The mean ratings of the most common associations from Phase 2 were analyzed qualitatively to assess the number of negatively rated associations (i.e., mean rating $<4$ ) for each label. A chi-square test was used to determine whether the proportion of the number of negatively rated associations for the different labels differed than what would be expected by chance.

Quantitative analyses. To address whether associations toward the different labels were significantly different from neutral, mean ratings were compared with a neutral rating of " 4 " using a series of one-sample $t$-tests. To address whether mean ratings between the labels differed, a $1 \times 8$ repeatedmeasures analysis of variance (ANOVA) was conducted. Follow-up pairwise comparisons were conducted using dependent-group $t$-tests.

Exploratory analyses. Specific associations. To further investigate the common association person for "a person not on the autism spectrum", and "a person without a disability," the association person and the similar association human were counted for all labels. Then chi-square analyses were conducted to determine whether the odds of generating the association person or human differed depending on the label.

Connection to autism and disability. To address whether mean ratings between labels differed depending on participants' connection to either autism or disability, two $2 \times 8$ repeated-measures ANOVAs were conducted. Follow-up comparisons were conducted using independent-group $t$-tests.

Connection to either autism or disability was defined as no or yes (either having a close friend or family member on the autism spectrum/disability or self-identifying as autistic/ disabled) because of the relatively few number of participants who self-identified as autistic/disabled as given in Table 1.

Power analysis and $\alpha$-level. With an $\alpha=0.05$ and power $=0.80$, the sample size needed to detect an effect size of 0.30 for a two-tailed dependent-groups $t$-test (i.e., the simplest within-group comparison) is 90 (GPower $3^{36}$ ); therefore, we aimed for $\sim 100$ participants per phase in each of the final samples. Furthermore, a conservative $\alpha=0.001$ was used to correct for multiple comparisons on all post hoc tests.

\section{Results}

Phase 1

A brief qualitative analysis was conducted to examine associations in common from Phase 1 (see Table 2) for the different labels in the two samples. Overall, there was a large overlap in the associations generated by the two samples suggesting moderate agreement. The two samples generated four associations in common for "a person with a disability," five associations in common for "a person without a disability," six associations in common for "a person on the autism spectrum," and seven associations in common for the remaining five labels.

"A person not on the autism spectrum" and "a person without a disability" shared the greatest number of associations (undergraduate sample $[n=6]$ : able-bodied, average, 


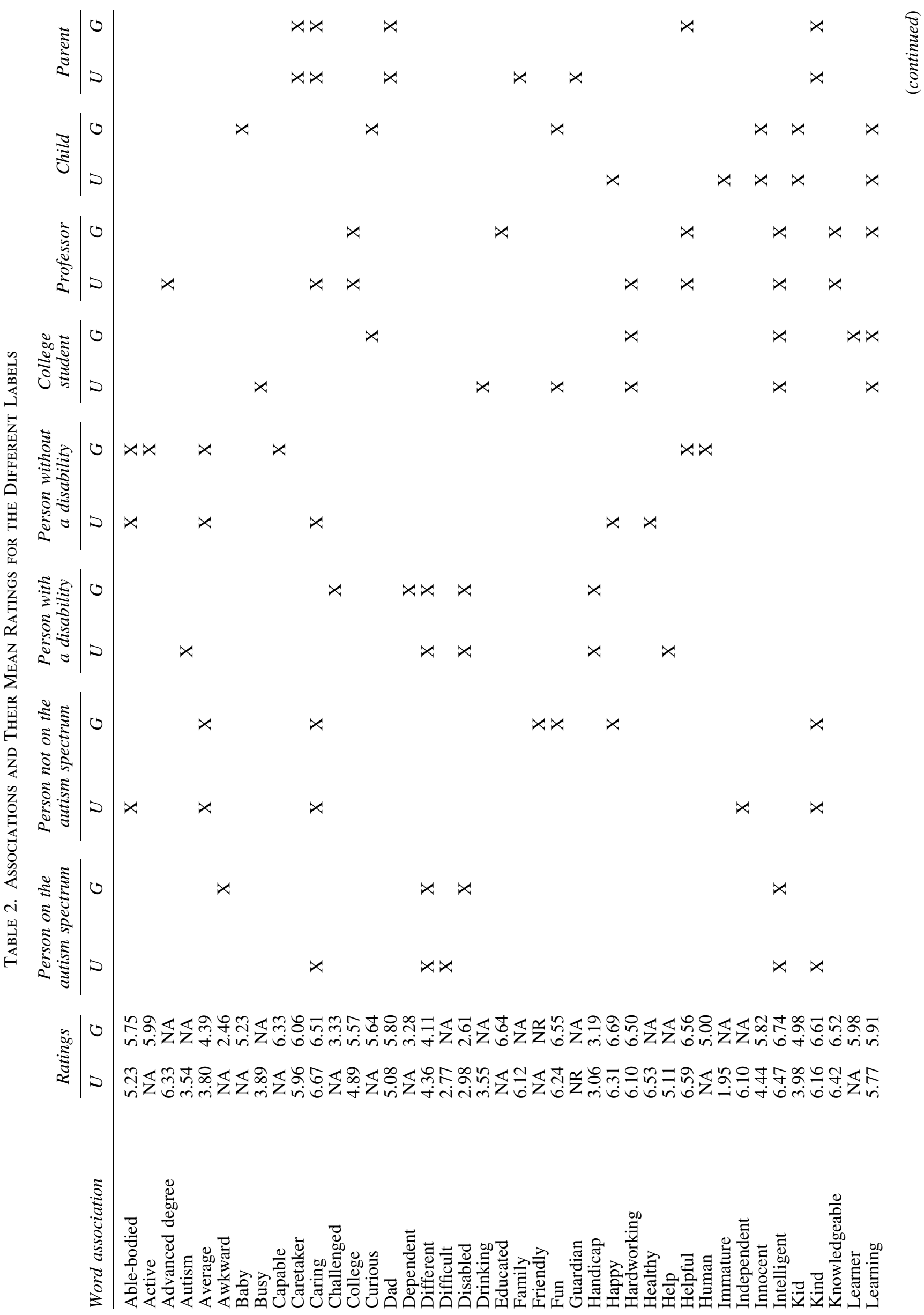




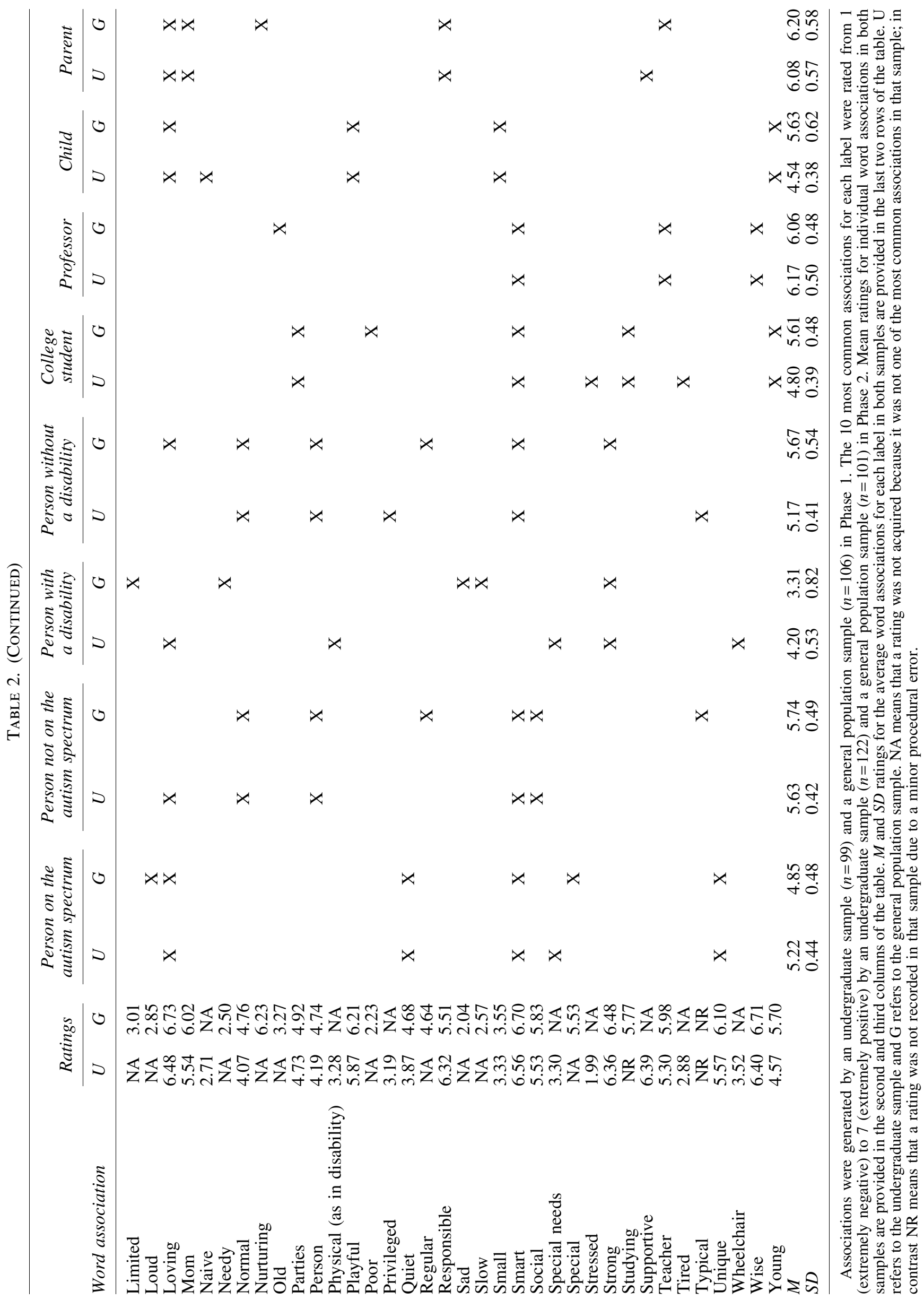


caring, normal, person, and social; general population sample $[n=5]$ : average, normal, person, regular, and smart). "A person on the autism spectrum" and "a person with a disability" shared some associations in common (undergraduate sample [ $n=3]$ : different, loving, and special needs; general population sample [ $n=2]$ : different and disabled), which was similar to other pairs of labels (undergraduate sample range: $0-4$; general population sample range: $0-3$ ).

\section{Phase 2}

Qualitative analysis. A brief qualitative analysis was conducted to examine the negatively rated associations in Phase 2 (see Table 2) for the different labels in the two samples. "A person with a disability" had the highest number of negatively rated associations (undergraduate sample [ $n=6]$ : autism, disabled, handicap, physical [as in disability], special needs, and wheelchair; general population sample $[n=8]$ : challenged, dependent, disabled, handicap, limited, needy, sad, and slow). "A person on the autism spectrum" had some negatively rated associations (undergraduate sample [n=3]: difficult, quiet, and special needs; general population sample [ $n=3]$ : awkward, disabled, and loud). This fell within the range of negatively rated associations for other labels for the undergraduate sample (range: 0-4) but was higher than all other labels for the general population sample (range: $0-1$ ). The proportion of negative associations across labels was significantly different than what was expected by chance [undergraduate sample: $\chi^{2}(7)=16.06, p=0.02$; general population sample, $\left.\chi^{2}(7)=36.80, p<0.001\right]$.

Quantitative analysis. Only "a person with a disability" $[t(100)=-8.38, p<0.001, d=-0.83, M=3.31, S D=0.82]$ in the general population sample was rated as having significant negative associations; all other labels in both samples were rated as having significant positive associations [undergraduate sample: minimum $t(121)=4.18, p<0.001, d=0.38$ for "a person with a disability," $M=4.20, S D=0.53$ ] as given in Table 2 .

However, as given in Table 2, mean ratings varied by label in both samples [undergraduate sample: $F(7,847)=574.62$, $p<0.001, \eta^{2}$ partial $=0.83$; general population sample: $F(7$, $\left.700)=646.89, p<0.001, \eta_{\text {partial }}^{2}=0.87\right]$. The associations of "a person with a disability" were rated more negatively than all other labels in both samples (all $p s<0.001$ ). The associations of "a person on the autism spectrum" were rated more negatively than all other labels, except for "a person with a disability," in the general population sample [all $p s<0.001$; minimum $t(100)=-17.84, \quad p<0.001, d=-1.77$ for " a child"]. The associations of "a person on the autism spectrum" were only rated more negatively than "a person not on the autism spectrum" $[t(121)=-13.08, p<0.001, d=-1.18]$, "a parent" $[t(121)=-21.23, p<0.001, d=-1.92]$, and "a professor" $[t(121)=-25.76, p<0.001, d=-2.33]$ in the undergraduate sample. However, undergraduate students rated the words autism and disabled, two of the most common associations for "a person with a disability," as significantly negative [autism: $t(121)=-4.81, p<0.001, d=-0.44$; disabled: $t(121)=-11.47, p<0.001, d=-1.04]$.

\section{Exploratory analyses}

Specific associations. The frequency of the associations person and human is presented in Table 3 . The odds of generating the association person or human differed depending on the label in both the undergraduate sample [person: $\chi^{2}(7)=46.42, p<0.001$; human: $\left.\chi^{2}(7)=30.32, p<0.001\right]$ and the general population sample [person: $\chi^{2}(7)=47.19$, $p<0.001$; human: $\left.\chi^{2}(7)=41.14, p<0.001\right]$. The associations person and human rarely or never occurred for "a college student," "a professor," "a child," or "a parent" in both samples. In contrast, these associations tended to be more likely in "a person without a disability" or "a person not on the autism spectrum" than "a person with a disability" or "a person on the autism spectrum" - a pattern that failed to reach significance in the undergraduate sample [person: $\chi^{2}(3)=5.02, p=0.17$; human: $\left.\chi^{2}(3)=2.15, p=0.54\right]$ and approached significance in the general population sample [person: $\chi^{2}(3)=14.81, p=0.002$; human: $\chi^{2}(3)=8.01$, $p=0.05]$.

Connection to autism and disability. Connection to autism did not have an effect on mean ratings [undergraduate sample: $F(1,120)=0.11, p=0.74, \eta^{2}$ partial $=0.001$; general population sample: $\left.F(1,99)=1.48, p=0.23, \eta_{\text {partial }}^{2}=0.02\right]$ and did not interact with the label on the mean ratings in either sample [undergraduate sample: $F(5,600)=0.57$, $p=0.73, \eta_{\text {partial }}^{2}=0.005$; general population sample: $F(5$, $\left.495)=0.58, p=0.90, \eta_{\text {partial }}^{2}=0.003\right]$. However, connection to disability did interact with the label on the mean ratings in the undergraduate sample $\left[F(5,600)=2.36, p=0.04, \eta_{\text {par- }}^{2}\right.$ tial $=0.02$ ]. All other main effects [undergraduate sample: $F(1,120)=0.07, p=0.79, \eta^{2}$ artial $=0.001$; general population sample: $\left.F(1,99)=0.02, p=0.89, \eta_{\text {partial }}^{2}<0.001\right]$ and interactions with connection to disability [general population: $\left.F(5,495)=0.58, p=0.72, \eta_{\text {partial }}^{2}=0.006\right]$ were not significant. Despite a significant interaction in the undergraduate sample, connection to disability did not affect mean ratings for any individual label [maximum $t(120)=1.66, p=0.10$ for "a parent"].

\section{Discussion}

Explicit attitudes toward the autism spectrum, in particular, and disability, in general, were mixed. Only "a person with a disability" in the general population sample was rated

Table 3. Frequency of Associations Person and Human from Phase 1 fOr Each Label in Two Samples

\begin{tabular}{|c|c|c|c|c|}
\hline \multirow[b]{2}{*}{ Label } & \multicolumn{2}{|c|}{$\begin{array}{l}\text { Undergraduates } \\
\mathrm{n}=99\end{array}$} & \multicolumn{2}{|c|}{$\begin{array}{c}\text { General } \\
\text { population } \\
\mathrm{n}=106\end{array}$} \\
\hline & Person & Human & Person & Human \\
\hline $\begin{array}{l}\text { Person on the autism } \\
\text { spectrum }\end{array}$ & 5 & 6 & 4 & 4 \\
\hline $\begin{array}{l}\text { Person not on the autism } \\
\text { spectrum }\end{array}$ & 13 & 6 & 15 & 10 \\
\hline Person with a disability & 6 & 10 & 3 & 7 \\
\hline $\begin{array}{l}\text { Person without } \\
\text { a disability }\end{array}$ & 12 & 7 & 14 & 15 \\
\hline College student & 0 & 0 & 2 & 1 \\
\hline Professor & 0 & 1 & 2 & 2 \\
\hline Child & 0 & 0 & 1 & 0 \\
\hline Parent & 0 & 0 & 1 & 1 \\
\hline
\end{tabular}


as having significant negative associations. Both samples generated the most negative associations for "a person with a disability," but the number of negative associations generated for "a person on the autism spectrum" was similar to the number of negative associations generated for other labels. However, associations of "a person with a disability" were rated more negatively than all other types of people in both samples and the associations of "a person on the autism spectrum" were rated as second most negative in the general population sample. Furthermore, the generated associations disabled and autism for "a person with a disability" were rated negatively in the undergraduate sample. Collectively, these findings suggest that undergraduate students and general population adults may have explicit negative associations with both the autism spectrum in particular and to disability more generally. This negative bias appears to be strongest in the general population sample, especially toward disabled people, and highlights the need for research in noncollege samples.

In both samples, person was one of the most common associations in response to "a person not on the autism spectrum" and "a person without a disability" but not "a person on the autism spectrum" or "a person with a disability" or any of the other four comparison labels. Exploratory analyses showed this pattern differed from what would be expected by chance and was similar to how often human was generated as an association for the different labels. Participants may have generated associations for the different labels by thinking of them as pairs, and the personhood of "a person without a disability" and "a person not on the autism spectrum" became salient only because they were paired with "a person with a disability" or "a person on the autism spectrum," suggesting that people may dehumanize autistic ${ }^{37}$ and disabled people. ${ }^{38}$ Participants may also have been primed to think of personhood because the word person appeared in the labels for "a person on the autism spectrum," "a person not on the autism spectrum," "a person with a disability," and "a person without a disability," but not in the other four labels. Additional research is recommended to understand why person (and to a lesser extent human) is a more common association of "a person without a disability" and "a person not on the autism spectrum."

Both samples included individuals with a connection to autism and disability, including some individuals who selfidentified as autistic and/or disabled, which suggests that at least half of the samples had some knowledge of autism and disability. One interesting question is whether people without a connection to autism or disability and people with a connection to autism or disability, including individuals who self-identify as autistic or disabled, differ in their explicit attitudes toward autism and disability. Although exploratory analyses attempted to examine this question, this was not the purpose of this study and additional research would be needed to recruit members from each group. More specifically, it was only possible to analyze the mean ratings separately for groups with or without a connection to autism and disability. Another question would be whether a connection to autism and disability affects self-generated associations and the valence of those self-generated associations.

This study used a novel method to investigate explicit associations of autism and disability. More specifically, the approach was novel because participants self-generated the associations and because the multiple labels permitted comparison of explicit attitudes toward autism, disability, and other groups. Future research could modify the procedure in an effort to replicate the findings. For example, participants were asked to "list the first 10 words (e.g., synonyms, adjectives, or other descriptors) that come to mind" and as a result, participants generated multiple parts of speech including adjectives and nouns. Similarly, participants may have had more difficulty generating associations for "a person not on the autism spectrum" and "a person without a disability" than other labels because they relied on vague descriptors. Future research could limit word associations to adjectives to increase the likelihood that associations are more descriptive. In addition, given the overlap in associations between labels (e.g., loving; see Table 2), analyses could be restricted to unique associations. Finally, future research could consider whether the terminology used to describe people affects the associations generated to describe them. More specifically, the more neutral wording "a person on the autism spectrum" was used; however, word associations for "an autistic person" (i.e., identity-first language) could be compared with word associations for "a person with autism" (i.e., person-first language) as person-first language may be stigmatizing. ${ }^{39}$

These findings may not extend to a more diverse population as both samples had relatively high education levels (i.e., at least some college), were primarily White non-Hispanic, and were living in the United States. In addition, the college sample had more females that is common in noninvasive research, ${ }^{40}$ but was specifically controlled for in the general population sample. Furthermore, participants may have generated more positive associations for "a person on the autism spectrum" and "a person with a disability" due to the threat of social desirability bias in assessing attitudes. ${ }^{35} \mathrm{~A}$ social desirability bias is of particular concern in the college student sample, especially given their overall positive associations in response to "a person of the autism spectrum" but negative rating of the word autism. Although several methods were used to minimize social desirability bias, including having participants generate and rate words in separate studies and using two separate samples to confirm the results (e.g., there was moderate agreement for the most common associations between samples), it is not possible to rule out the role of social desirability bias. For example, the word association unique for "a person on the autism spectrum" may have been a more socially desirable or positive term to communicate a negative impression of autistic individuals.

Ultimately, the possible explicit negative associations with the autism spectrum and disability are concerning because they reflect people's conscious and controllable evaluations. Furthermore, these results are contrary to previous studies reporting clear positive explicit attitudes toward autism ${ }^{4}$ and disability more generally. ${ }^{41}$ These results highlight the imperative need to confront ableism through attitudes to improve acceptance and inclusion of autistic and disabled people in society.

\section{Acknowledgments}

We thank Sarah Wonsidler for her assistance with data collection and Nicole Dalasio for her feedback on a draft of this article. 


\section{Authors' Contributions}

J.L.S. conceived the study, performed statistical analysis, and drafted the article; J.L.S. and T.G.M. designed the study and interpreted the data; and T.G.M. coordinated the study and edited the article. Both authors reviewed and approved the article before submission.

\section{Disclaimer}

This article has been submitted solely to Autism in Adulthood and is not published, in press, or under submission elsewhere.

\section{Author Disclosure Statement}

No competing financial interests exist.

\section{Funding Information}

The authors thank Athena Institute for funding this project.

\section{References}

1. Dillenburger K, Jordan JA, McKerr L, Devine P, Keenan M. Awareness and knowledge of autism and autism interventions: A general population survey. Res Autism Spectr Disord. 2013;7(12):1558-1567.

2. Durand-Zaleski I, Scott J, Rouillon F, Leboyer M. A first national survey of knowledge, attitudes and behaviours towards schizophrenia, bipolar disorders and autism in France. BMC Psychiatry. 2012;12:128.

3. Tipton LA, Blacher J. Brief report: Autism awareness: Views from a campus community. J Autism Dev Disord. 2014;44(2):477-483.

4. White D, Hillier A, Frye A, Makrez E. College students' knowledge and attitudes towards students on the autism spectrum. J Autism Dev Disord. 2016;49(7):2699-2705.

5. Brown L. Dear well-meaning stranger. In: Autistic Self Advocacy Network, Bascom J, eds. And Straight on Till Morning: Essays on Autism Acceptance. Washington, DC: The Autistic Press; 2013.

6. Gladstone K. It's autism Sunday (pity party at church). In: Autistic Self Advocacy Network, Bascom J, eds. And Straight on Till Morning: Essays on Autism Acceptance. Washington, DC: The Autistic Press; 2013.

7. Kassiane S. Acceptance vs. awareness. In: Autistic Self Advocacy Network, Bascom J, eds. And Straight on Till Morning: Essays on Autism Acceptance. Washington, DC: The Autistic Press; 2013.

8. Rosa SDR. All done autism awareness. In: Autistic Self Advocacy Network, Bascom J, eds. And Straight on Till Morning: Essays on Autism Acceptance. Washington, DC: The Autistic Press; 2013.

9. Dillenberger K, McKen L, Jordan JA, Devine P, Keenan M. Creating an inclusive society... How close are we in relation to autism spectrum disorders? A general population survey. J Appl Res Intellect Disabil. 2015;28(4):330-340.

10. Maroto M, Pettinicchio D. Twenty-five years after the ADA: Situating disability in America's system of stratification. Disabil Stud Q. 2015;35(3):4927.

11. Chambres P, Auxiette C, Vansingle C, Gil S. Adult attitudes toward behaviors of a six-year-old boy with autism. $J$ Autism Dev Disord. 2008;38(7):1320-1327.
12. Iobst E, Nabors L, Rosenzweig K, et al. Adults' perceptions of a child with autism. Res Autism Spectr Disord. 2009; 3(2):401-408.

13. Harnum D, Duffy J, Ferguson DA. Adults' versus children's perceptions of a child with autism or Attention Deficit Hyperactivity Disorder. J Autism Dev Disord. 2007; 37(7):1337-1343.

14. Park M, Chitiyo M, Choi YS. Examining pre-service teachers' attitudes towards children with autism in the USA. J Res Spec Educ Needs. 2010;10(2):107-114.

15. Campbell JM. Brief report: Reliability and validity of the Shared Activities Questionnaire as a measure of middle school students' attitudes toward autism. J Autism Dev Disord. 2008;38(8):1598-1604.

16. Campbell JM, Ferguson JE, Herzinger CV, Jackson JN, Marino CA. Combined descriptive and explanatory information improves peers' perceptions of autism. Res Dev Disabil. 2004;25(4):321-339.

17. Morton JF, Campbell JM. Information source affects peers' initial attitudes toward autism. Res Dev Disabil. 2008; 29(3):189-201.

18. Reiter S, Vitani T. Inclusion of pupils with autism: The effect of an intervention program on the regular pupils' burnout, attitudes and quality of mediation. Autism. 2007; 11(4):321-333.

19. Swaim KF, Morgan SB. Children's attitudes and behavioral intentions toward a peer with autistic behaviors: Does a brief educational intervention have an effect? J Autism Dev Disord. 2001;31(2):195-205.

20. Hines M, Balandin S, Togher L. Buried by autism: Older parents' perceptions of autism. Autism. 2012;16(1):15-26.

21. Brosnan M, Mills E. The effect of diagnostic labels on the affective responses of college students towards peers with "Asperger's Syndrome" and "Autism Spectrum Disorder." Autism. 2016;20(4):388-394.

22. Butler RB, Gillis JM. The impact of labels and behaviors on the stigmatization of adults with Asperger's Disorder. $J$ Autism Dev Disord. 2011;41(6):741-749.

23. Gardiner E, Iarocci G. Students with autism spectrum disorder in the university context: Peer acceptance predicts intention to volunteer. J Autism Dev Disord. 2014;44(5): 1008-1017.

24. Matthews NL, Ly AR, Goldberg WA. College students' perceptions of peers with autism spectrum disorder. $J$ Autism Dev Disord. 2015;45(1):90-99.

25. Nevill REA, White SW. College students' openness toward autism spectrum disorders: Improving peer acceptance. $J$ Autism Dev Disord. 2011;41(12):1619-1628.

26. Campbell JM. Middle school students' response to the selfintroduction of a student with autism: Effects of perceived similarity, prior awareness, and educational message. Remedial Spec Educ. 2007;28(3):163-173.

27. Gillespie-Lynch K, Brooks PJ, Someki F, et al. Changing college students' conceptions of autism: An online training to increase knowledge and decrease stigma. J Autism Dev Disord. 2015;45(8):2553-2566.

28. Obeid R, Daou N, DeNigris D. A cross-cultural comparison of knowledge and stigma associated with autism spectrum disorder among college students in Lebanon and the United States. J Autism Dev Disord. 2015;45(11):3520-3536.

29. Staniland JJ, Byrne MK. The effects of a multi-component higher-functioning autism anti-stigma program on adolescent boys. J Autism Dev Disord. 2013;43(12):28162829. 
30. Sasson NJ, Morrison KE. First impressions of adults with autism improve with diagnostic disclosure and increased autism knowledge of peers. Autism. 2019;23(1):50-59.

31. Sasson NJ, Faso DJ, Nugent J, et al. Neurotypical peers are less willing to interact with those with autism based on thin slice judgements. Sci Rep. 2017;7:40700.

32. Friedman C, Owen AL. Defining disability: Understandings of and attitudes towards ableism and disability. Disabil Stud Q. 2017;37(1):4545.

33. Gernsbacher MA, Raimond AR, Stevenson JL, Boston JS, Harp B. Do puzzle pieces and autism puzzle piece logos evoke negative associations? Autism. 2018;22(2):118-125.

34. Wilson MC, Scior K. Attitudes towards individuals with disabilities as measured by the Implicit Association Test: A literature review. Res Dev Disabil. 2014;35(2):294-321.

35. Antonak RF, Livneh H. Measurement of attitudes towards persons with disabilities. Disabil Rehabil. 2000;22(5):211224.

36. Faul F, Erdfelder E, Lang A-G, Buchner A. G*Power 3: A flexible statistical power analysis program for the social, behavioral, and biomedical sciences. Behav Res Methods. 2007;39(2):175-191.

37. Cage E, Di Monaco J, Newell V. Understanding, attitudes and dehumanization towards autistic people. Autism. 2019; 23(6):1373-1383.
38. Capozza D, Di Bernardo GA, Falvo R, Vianello R, Calò L. Individuals with intellectual and developmental disabilities: Do educators assign them a fully human status? J Appl Soc Psychol. 2016;46(9):497-509.

39. Gernsbacher MA. Editorial perspective: The use of personfirst language in scholarly writing may accentuate stigma. $J$ Child Psychol Psychiatry. 2017;58(7):859-861.

40. Rosenthal R, Rosnow RL. Artifacts in Behavioral Research. Oxford, United Kingdom: Oxford University Press; 2009.

41. Wilson MC, Scior K. Implicit attitudes towards people with intellectual disabilities: Their relationship with explicit attitudes, social distance, emotions, and contact. PLoS One. 2015;10(9):e0137902.
Address correspondence to: Jennifer L. Stevenson, PhD Department of Psychology Ursinus College 601 East Main Street Collegeville, PA 19426

Email: jstevenson@ursinus.edu 